\title{
HTLV-I Production Based on Activation of Integrin/Ligand Signaling in HTLV-I-Infected T Cell Lines Derived from HAM/TSP Patients
}

Running title: Integrin/Ligand Signaling in HTLV-I production

Key words: HTLV-I, HAM/TSP, small GTPase, integrin

Naomi Fukushima ${ }^{\mathrm{a}}$, Tatsufumi Nakamura ${ }^{\mathrm{a}}$, Yoshihiro Nishiura ${ }^{\mathrm{b}}$, Hiroaki Ida ${ }^{\mathrm{b}}$, Toshiyuki Aramaki ${ }^{\mathrm{b}}$, Katsumi Eguchi ${ }^{\mathrm{b}}$

\footnotetext{
${ }^{a}$ Department of Molecular Microbiology and Immunology

${ }^{\mathrm{b}}$ First Department of Internal Medicine

Graduate School of Biomedical Sciences, Nagasaki University, Japan
}

Corresponding author: Tatsufumi Nakamura, M.D.

Department of Molecular Microbiology and Immunology, Graduate School of

Biomedical Sciences, Nagasaki University, 1-12-4 Sakamoto, Nagasaki 852-8523, Japan

Phone: +81-95-819-7265

Fax: +81-95-849-7270

E-mail: tatsu@net.nagasaki-u.ac.jp 


\section{Abstract}

Objective: Activity of integrin/ligand signaling leading to activation of small GTPases might regulate the efficiency of cell-to-cell spread of human T lymphotropic virus type I (HTLV-I) through the virological synapse. We compared both activity of small GTPases and involvement of integrin/ligand signaling in extracellular release of HTLV-I virions between each three HTLV-I-infected T cell lines derived from HTLV-I-associated myelopathy/tropical spastic paraparesis (HAM/TSP) patients and from other origins as a control. Methods: activity of small GTPases (Rho, Rac, and Cdc42) was analyzed by pull-down assay with suppressive effect of both HTLV-I production and HTLV-I tax mRNA expression by anti-integrin blocking antibodies. Results: All small GTPases were strongly activated in all cell lines derived from HAM/TSP patients, but not in control cell lines except one cell line. Treatment of all cell lines derived from HAM/TSP patients, but not all control cell lines, with anti-integrin blocking antibodies significantly suppressed the level of HTLV-I p19 antigen in culture supernatants without down-regulation of HTLV-I tax mRNA expression. Conclusion: Significant involvement with integrin/ligand signaling in extracellular release of HTLV-I virions in cell lines derived from HAM/TSP patients suggests that HTLV-I-infected cells in HAM/TSP patients have potential for the efficient spread of HTLV-I to uninfected cells. 


\section{Introduction}

In general, human T lymphotropic virus type I (HTLV-I) infects cells through cell-to-cell contact but not cell-free virions in vivo [1, 2]. Recently, Igakura et al. clearly demonstrated that HTLV-I spreads to cells through the virological synapse, which is composed of adhesion molecules such as integrin and ligand, by polarization of the cytoskeleton, such as the microtubule-organizing center (MTOC) [3]. Integrin/ligand signaling induces the activation of small GTPases, such as Rho, Rac, and Cdc42, and activation of this signaling pathway is involved in the rearrangement of cytoskeletal components [4, 5]. Of them, Rac and Cdc42 regulate both actin and microtube cytoskeletons, including actin polymerization, cell polarity, and the reorientation of MTOC [6-8]. Therefore, activation of adhesion molecule signaling induced by the interaction between integrin and its ligand activates MTOC polarization mediated by the activation of both Rac and Cdc42, and the degree of activity of these signaling pathways might determine the efficiency of cell-to-cell spread of HTLV-I.

HTLV-I-associated myelopathy/tropical spastic paraparesis (HAM/TSP) is a chronic progressive myelopathy characterized by bilateral pyramidal tract involvement with sphincteric disturbances [9]. The primary pathological feature of HAM/TSP is chronic inflammation of the spinal cord characterized by perivascular cuffing and parenchymal infiltration of mononuclear cells [10]. At the present time, although it is supposed that the destruction of the surrounding nervous tissue induced by the interaction between HTLV-I-infected CD4 ${ }^{+}$T cells and HTLV-I-specific CD8 ${ }^{+}$cytotoxic T cells, so called "bystander mechanisms”, is probably critical in the immunopathological process 
leading to HAM/TSP [11,12], the exact mechanisms underlying chronic myelitis caused by HTLV-I infection are still unknown. We previously reported significantly increased activity of adherence to, and subsequent transmigration through human endothelial cells (EC) of activated CD4 ${ }^{+}$T cells with heightened lymphocyte function antigen-1 (LFA-1, $\alpha \mathrm{L} \beta 2$ ) expression in the peripheral blood T cells of HAM/TSP patients [13, 14], suggesting that signaling induced by integrin/ligand interaction is up-regulated in peripheral blood $\mathrm{CD}^{+}{ }^{+} \mathrm{T}$ cells of HAM/TSP patients. Therefore, activation of this signaling itself leading to the activation of small GTPases, as mentioned above, might be related to the efficient cell-to-cell spread of HTLV-I involved in the induction of aberrant immunological functions in HTLV-I-infected cells of the peripheral blood in HAM/TSP patients.

From these viewpoints, we first analyzed the activity of small GTPases, such as Rho, Rac and Cdc42, in three HTLV-I-infected T cell lines derived from HAM/TSP patients, compared to three HTLV-I-infected T cell lines derived from other origins as controls. When considering cell-to-cell spread of HTLV-I, the extracellular release of HTLV-I virions, that is, HTLV-I production from HTLV-I-infected cells, might be one of the indicators of cell-to-cell spread. If cell-to-cell spread of HTLV-I is regulated by integrin/ligand activity, its efficacy might depend on the release of HTLV-I virions from HTLV-I-infected cells under integrin/ligand interaction. Therefore, secondly, in order to analyze the degree of the involvement of integrin/ligand interaction in the release of HTLV-I virions, we investigated the suppressive effect of HTLV-I production by anti-integrin blocking antibodies, such as anti- $\alpha \mathrm{L} \beta 2$ and very late-activating antigen-4 
(VLA-4, $\alpha 4 \beta 1$ ) antibody, in HTLV-I-infected T cell lines derived from HAM/TSP patients, compared to control cell lines.

\section{Materials and Methods}

\section{Cell lines}

HTLV-I-infected T cell lines derived from the cerebrospinal fluid of three different HAM/TSP patients (HCT-1, HCT-4, and HCT-5) were used [15]. These cell lines were interleukin(IL)-2-dependent and maintained in RPMI 1640 containing 20\% fetal bovine serum (FBS) supplemented with 100 units/ml of recombinant human IL-2 (kindly provided by Shionogi, Japan). HTLV-I-infected T cell lines (TL-Su, TCL-Kan, and C91/PL) were used as control cell lines. TL-Su, TCL-Kan, and C91/PL were HTLV-I producing cell lines derived from an HTLV-I asymptomatic carrier, adult peripheral blood, and cord blood, respectively [16-18]. These cell lines were maintained in RPMI 1640 containing $10 \%$ FBS. From flow cytometric analysis of the expression of integrins ( $\alpha \mathrm{L} \beta 2$ and $\alpha 4 \beta 1$ ) and ligands (ICAM-1 and VCAM-1) in all cell lines, the expression of all integrins/ligands was confirmed except for the weak expression of $\alpha 4 \beta 1$ on control cell lines. Our data, such as ICAM-1 and VCAM-1 expression in HTLV-I-infected T cell lines, are consistent with the previous reports [19, 20].

\section{Small GTPase pull-down assay}

After each cell line was seeded at $2 \times 10^{5}$ cells/ml, and cultured for 15 hrs, cells were collected and lysed by ice-cold cell lysis buffer. Pull-down assays for Rho-, Rac- 
and Cdc42-GTP were performed using Rho, Rac and Cdc42 activation biochem kits, respectively, according to the manufacturer's instructions (Cytoskeleton, Inc., Denver, CO). Briefly, after cell lysates were clarified by centrifugation, equal amounts of lysates (1 mg per cell line) were incubated with $45 \mu$ g of Rhotekin-RBD beads for Rho, and 20 $\mu \mathrm{g}$ of PAK-PBD beads for Rac and Cdc42 for $1 \mathrm{hr}$ on a rotator at $4^{\circ} \mathrm{C}$. The beads were washed, and bound Rho-, Rac-, Cdc42-GTP was subjected to Western blot analysis.

\section{Blocking study by anti-integrin antibodies}

Each cell line was seeded at a concentration of $2 \times 10^{5}$ cells/ml, and cultured in the presence of $20 \mu \mathrm{g} / \mathrm{ml}$ of both anti-CD11a/LFA-1 ( $\alpha \mathrm{L} \beta 2$ ) blocking antibody (clone; HI111, BD Biosciences) and anti- $\alpha 4 / C D 49 d(\alpha 4 \beta 1)$ blocking antibody (clone; 2B4, R\&D Systems) or $40 \mu \mathrm{g} / \mathrm{ml}$ of control (irrelevant) antibody (clone; MOPC-21, BioLegend) for 15 hrs. In the time course study, the culture supernatant was collected at 3,6 , and 15 hrs after the beginning of culture. The culture medium was centrifuged at $2500 \mathrm{rpm}$ for $10 \mathrm{~min}$ and the supernatant was stored at $-40^{\circ} \mathrm{C}$ until use. In some experiments, cells were collected at 6 and 15 hrs after the beginning of culture for quantitative RT-PCR study.

\section{Quantitative RT-PCR analysis}

For quantitative analysis of HTLV-I tax mRNA expression, real-time quantitative RT-PCR was performed in a Light-Cycler FastStart DNA Master (Roche Diagnostics, Mannheim, Germany) based on general fluorescence detection with SYBR Green as 
described previously [21]. Briefly, after total cellular RNA was extracted, cDNA was synthesized from RNA treated with RQ1 RNase-free DNase using Oligo (dT) 12-18 primer (Promega, Madison, WI) by M-MLV reverse transcriptase (Promega). The following oligonucleotide primers were used to amplify a 247 bp fragment of HTLV-I tax (forward; AAACAGCCCTGCAGAGATACAAAGT, reverse;

ACTGTAGAGCTGAGCCGATAACG) and a 236 bp fragment of $\beta$-actin (forward; GACGAGGCCCAGAGCAAGAGAG, reverse; ACGTACATGGCTGGGGTGTTG) respectively. HTLV-I tax mRNA expression was evaluated by normalization to $\beta$-actin.

\section{Measurement of HTLV-I p19 antigen level in culture supernatants}

The level of HTLV-I p19 antigen in culture supernatants was measured using the RETROtek HTLV p19 antigen enzyme-linked immunosorbent assay (ELISA) kit following the manufacturer's instructions (Zeptometrix Corporation, Buffalo, NY). The amount of HTLV-I p19 antigen was determined in duplicate. The minimum measurable level of HTLV-I p19 antigen was 25.0 pg/ml.

\section{Statistical analysis}

Student s t-test was used for statistical analysis. Differences were considered significant at $p<0.05$.

\section{Results}

Small GTPases, particularly Cdc42, are strongly activated in HTLV-I-infected T 
cell lines derived from HAM/TSP patients

As shown in Figure 1, all bound Rho-, Rac-, and Cdc42-GTP were detected in all three HTLV-I-infected T cell lines derived from HAM/TSP patients. However, bound Rho-, Rac-, and Cdc42-GTP were detected in only TL-Su of control cell lines. In TL-Su, although the amounts of both bound Rho- and Rac-GTP seemed to be almost the same as in cell lines derived from HAM/TSP patients, the amount of bound Cdc42-GTP was obviously lower than in cell lines derived from HAM/TSP patients, indicating that the degree of activation of Cdc42 is lower in TL-Su than in cell lines derived from HAM/TSP patients.

HTLV-I production from HTLV-I-infected T cell lines derived from HAM/TSP patients is significantly suppressed by treatment with anti-integrin blocking antibodies

As shown in Figure 2, the treatment of HCT-1, HCT-4, and HCT-5 with both anti- $\alpha \mathrm{L} \beta 2$ and - $\alpha 4 \beta 1$ blocking antibodies significantly suppressed the levels of HTLV-I p19 antigen in culture supernatants by about 80, 50, and 50\%, respectively. However, the same treatment of TL-Su, TCL-Kan, and C91/PL did not suppress the levels of HTLV-I p19 antigen in culture supernatant (Figure 2). In order to confirm that anti-integrin blocking antibodies do not induce the cytotoxic effects for HTLV-I-infected cell lines, we compared the cell viabilities between anti-integrin blocking antibodies and control antibody-treated cells in each cell line by MTS assay simultaneously. As the results, there were no significant differences between both 
groups in each cell line (data not shown). These data indicate that HTLV-I production from cell lines derived from HAM/TSP patients, but not control cell lines, is significantly down-regulated by the interruption of integrin/ligand signaling.

Treatment with anti-integrin blocking antibodies against HTLV-I-infected T cell lines derived from HAM/TSP patients does not induce the down-regulation of HTLV-I expression

The time course study in HCT-1 treated with anti- $\alpha \mathrm{L} \beta 2$ and - $\alpha 4 \beta 1$ blocking antibodies revealed that a suppressive effect in HTLV-I production was detected at $3 \mathrm{hrs}$ after the beginning of culture (Figure 3). In addition, as shown in Figure 4, there were no significant differences of HTLV-I tax mRNA expression at 6 and 15 hrs after the beginning of culture between treatment with anti- $\alpha \mathrm{L} \beta 2$ and - $\alpha 4 \beta 1$ blocking antibodies and control antibody in both HCT-1 and HCT-5. These results strongly suggest that the effect of treatment with anti-integrin blocking antibodies against HTLV-I-infected T cell lines derived from HAM/TSP patients is based on suppression of the extracellular release of HTLV-I virions, but not on the down-regulation of HTLV-I expression.

\section{Discussion}

In this report, we demonstrated that small GTPases, such as Rho, Rac and Cdc42, are strongly activated in all three HTLV-I-infected T cell lines derived from HAM/TSP patients. On the other hand, activation of these small GTPases was not detected in 
control cell lines except TL-Su. However, the degree of activation of Cdc42 was obviously lower in TL-Su than in all three cell lines derived from HAM/TSP patients although the activation of both Rho and Rac was almost the same as that of cell lines derived from HAM/TSP patients. HTLV-I-infected cells can interact with each other through integrins and ligands, which are expressed on cells themselves, in our culture systems. Therefore, these data suggest that HTLV-I-infected T cell lines derived from HAM/TSP patients have more activated status of small GTPases, which are the downstream targets of integrin/ligand signaling [ 5], than control cell lines. Interestingly, the interruption of integrin/ligand signaling in all three HTLV-I-infected T cell lines derived from HAM/TSP patients, but not all control cell lines, with anti-integrin blocking antibodies, significantly suppressed HTLV-I production. It was suggested that these phenomena are based on down-regulation of the extracellular release of HTLV-I virions, but not HTLV-I expression as follows: 1) a suppressive effect on HTLV-I production of HCT-1 treated with anti-integrin blocking antibodies was detected at 3 hrs after the beginning of treatment; 2) there were no significant differences in the mRNA expression of HTLV-I tax in either HCT-1 or HCT-5 between control antibody-treated and anti-integrin blocking antibody-treated cells at 6 and 15 hrs after the beginning of treatment. These data might indicate that integrin/ligand signaling, which is the upstream of small GTPases, plays an important role in the extracelluar release of HTLV-I virions in HTLV-I-infected T cell lines derived from HAM/TSP patients. Thus, our data suggested that there are, at least, two distinct mechanisms in the extracellular release of HTLV-I virions in HTLV-I-infected cells, such as the system based on the 
activation of integrin/ligand signaling followed by activation of small GTPases or others. Interestingly, the degree of utilization of the mechanism of integrin/ligand signaling, which plays a crucial role in cell-to-cell spread of HTLV-I through the virological synapse, was higher in HTLV-I-infected T cell lines derived from HAM/TSP patients than in control cell lines during the extracellular release of HTLV-I virions, suggesting that HTLV-I-infected T cell lines derived from HAM/TSP patients have potential for efficient spread of HTLV-I to uninfected cells. However, we need to directly evaluate the efficiency of HTLV-I spread by using the coculture system of HTLV-I-infected T cell lines with non-infected T cell lines.

In this study, although Rho, Rac and Cdc42 were activated in TL-Su as same as all cell lines derived from HAM/TSP patients, the interruption of integrin/ligand signaling with anti-integrin blocking antibodies did not suppress HTLV-I production in this cell line. How can this discrepancy be explained? The relatively lower activation of Cdc42, compared to it in cell lines derived from HAM/TSP patients, might be involved. Indeed, Etienne-Manneville and Hall reported that Cdc42 plays an important role, rather than Rac or Rho, in the polarization of MTOC in integrin-mediated activated status in astrocytes [8]. Therefore, HTLV-I-infected T cell lines derived from HAM/TSP patients might have more polarized status of MTOC leading to the extracellular release of HTLV-I virions by utilization of the integrin/ligand signaling pathway although we did not actually analyze the polarized status of MTOC in this study. Our data support the previous results that the polarized condition of MTOC in HTLV-I-infected T cells might depend on Rac or Cdc42 rather than Rho activation [22]. 
As HTLV-I-infected cells can interact with each other through integrins and ligands in our culture systems as mentioned above, our data suggest the existence of another molecule, which activates integrin/ligand signaling, in HTLV-I-infected T cell lines derived from HAM/TSP patients. In this regard, we can confirm the activation of small GTPase Rap1, which is a potent stimulator of integrins as a complex with the regulator of adhesion and cell polarization enriched in lymphoid tissues (RAPL) and facilitates inside-out signaling [23], in all three HTLV-I-infected T cell lines derived from HAM/TSP patients (unpublished data). Although the exact mechanisms of how the activation of Rho, Rac and Cdc42 mediated by the activation of integrin/ligand signaling is induced in HTLV-I-infected T cell lines derived from HAM/TSP patients are still unknown, HTLV-I-infected T cell lines derived from HAM/TSP patients might have potential to easily activate small GTPases, particularly Cdc42, through integrin/ligand signaling leading to MTOC polarization. Therefore, in vivo, HTLV-I-infected cells in HAM/TSP patients might be under the preactivated status of MTOC polarization and this condition might induce the efficient cell-to-cell spread of HTLV-I by contact with non-infected T cells through the virological synapse. Further investigations are underway.

It is well known that high HTLV-I proviral load in the peripheral blood is the most important prerequisite in the development of HAM/TSP [24, 25]. The increased proliferation of HTLV-I-infected cells [26, 27], the relatively lower activity of HTLV-I-specific CD8 ${ }^{+}$cytotoxic T cells to HTLV-I-infected CD4 ${ }^{+}$T cells [25], and the active replication of HTLV-I [28, 29] etc., are proposed as reasons for the induction of 
high HTLV-I proviral load in HAM/TSP patients. Of them, the increased proliferation of HTLV-I-infected cells seems to play an important role mainly in the maintenance of high proviral load in the peripheral blood. However, the efficient cell-to-cell spread by activation of small GTPases in HTLV-I-infected cells also might partially function as one of the factors for the induction of high HTLV-I proviral load in peripheral blood of HAM/TSP patients, although our data presented in this report are based on the analyses in HTLV-I-infected T cell lines derived from HAM/TSP patients.

\section{Acknowledgements}

This work was supported in part by a Neuroimmunological Disease Research Committee Grant from the Ministry of Health, Labour and Welfare, Japan 


\section{References}

1 Okochi K, Sato H, Hinuma, Y: A retrospective study on transmission of adult $\mathrm{T}$ cell leukemia virus by blood transfusion: seroconversion in recipients. Vox Sang 1984;161:245-253.

2 Bangham CR: The immune control and cell-to-cell spread of human T-lymphotropic virus type 1. J Gen Virol 2003;84:3177-3189.

3 Igakura T, Stinchcombe JC, Goon PK, Taylor GP, Weber JN, Griffiths GM, Tanaka Y, Osame M, Bangham CR: Spread of HTLV-I between lymphocytes by virus-induced polarization of the cytoskeleton. Science 2003;299:1713-1716.

4 DeMali KA, Wennerberg K, Burridge K: Integrin signaling to the actin cytoskeleton. Curr Opin Cell Biol 2003;15:572-582.

5 Wittchen ES, van Buul JD, Burridge K, Worthylake RA: Trading spaces: Rap, Rac, and Rho as architects of transendothelial migration. Curr Opin Hematol 2005;12:14-21.

6 Daub H, Gevaert K, Vandekerckhove J, Sobel A, Hall A: Rac/Cdc42 and p65PAK regulate the microtubule-destabilizing protein stathmin through phosphorylation at serine 16. J Biol Chem 2001;276:1677-1680.

7 Palazzo AF, Joseph HL, Chen Y-J, Dujardin DL, Alberts AS, Pfister KK, Vallee RB, Gundersen GG: Cdc42, dynein, and dynactin regulate MTOC reorientation independent of Rho-regulated microtubule stabilization. Curr Biol 2001;11:1536-1541.

8 Etienne-Manneville S, Hall A: Integrin-mediated activation of Cdc42 controls cell 
polarity in migrating astrocytes through PKC $\zeta$. Cell 2001;106:489-498.

9 Osame M, Usuku K, Izumo S, Ijichi N, Amitani H, Igata A, Matsumoto M, Tara M: HTLV-I associated myelopathy, a new clinical entity. Lancet 1986;1:1031-1032.

10 Iwasaki Y: Pathology of chronic myelopathy associated with HTLV-I infection (HAM/TSP). J Neurol Sci 1990;96:103-123.

11 Ijichi S, Izumo S, Eiraku N, Machigashira K, Kubota R, Nagai M, Ikegami N, Kashio N, Umehara F, Maruyama I, Osame M: An autoaggressive process against bystander tissues in HTLV-I-infected individuals: a possible pathomechanism of HAM/TSP. Med hypotheses 1993;41:542-547.

12 Osame M: Pathological mechanisms of human T-cell lymphotropic virus type I-associated myelopathy (HAM/TSP). J Neurovirol 2002;8:359-364.

13 Ichinose K, Nakamura T, Nishiura Y, Nagasato K, Ohishi K, Watanabe H, Fujita A, Kurouji K, Tsujihata M, Nagataki S: Characterization of adherent T cells to human endothelial cells in patients with HTLV-I-associated myelopathy. J Neurol Sci 1994;122:204-209.

14 Ichinose K, Nakamura T, Nishiura Y, Tsujino A, Goto H, Shirabe S, Furuya T, Nagataki S: Characterization of T cells transmigrating through human endothelial cells in patients with HTLV-I-associated myelopathy. Immunobiol 1996/97;196:485-490.

15 Fukushima N, Nishiura Y, Nakamura T, Yamada Y, Kohno S, Eguchi K: Involvement of p38 MAPK signaling pathway in IFN- $\gamma$ and HTLV-I expression in patients with HTLV-I-associated myelopathy/tropical spastic paraparesis. J 
Neuroimmunol 2005;159:196-202.

16 Sugamura K, Fujii M, Kannagi M, Sakitani M, Takeuchi M, Hinuma Y:

Cell-surface phenotypes and expression of viral antigens of various human cell lines carrying human T-cell-leukemia virus. Int J Cancer 1984;34:221-228.

17 Yamamoto N, Okada M, Koyanagi Y, Kannagi M, Hinuma Y:

Transformation of human leukocytes by co-cultivation with an adult T-cell-leukemia-virus-producer cell line. Science 1982;217:737-739.

18 Popovic M, Lange-Wantzin G, Sarin PS, Mann D, Gallo RC: Transformation of human umbilical cord blood $\mathrm{T}$ cells by human $\mathrm{T}$-cell leukemia/lymphoma virus. Proc Nat Acad Sci USA 1983;80:5402-5406.

19 Fukudome K, Furuse M, Fukuhara N, Orita S, Imai T, Takagi S, Nagira M, Hinuma Y, Yoshie O: Strong induction of ICAM-1 in human T cells transformed by human T-cell-leukemia virus type 1 and depression of ICAM-1 or LFA-1 in adult T-cell-leukemia-derived cell lines. Int J Cancer 1992;52:418-427.

20 Valentin H, Lemasson I, Hamaia S, Cassé H, König S, Devaux C, Gazzolo L: Transcriptional activation of the vascular cell adhesion molecule-1 gene in $\mathrm{T}$ lymphotropic expressing human T-cell leukemia virus type 1 Tax protein. J Virol 1997;71:8522-8530.

21 Nishiura Y, Nakamura T, Fukushima N, Moriuchi R, Katamine S, Eguchi K: Increased mRNA expression of Th1-cytokine signaling molecules in patients with HTLV-I-associated myelopathy/tropical spastic paraparesis. Tohoku J Exp Med 2004;204:289-298. 
22 Nejmeddine M, Barnard AL, Tanaka Y, Taylor GP, Bangham CR: Human T-lymphotropic virus, type1, tax protein triggers microtubule reorientation in the virological synapse. J Biol Chem 2005;280:29653-29660.

23 Kinashi T, Katagiri K: Regulation of immune cell adhesion and migration by regulator of adhesion and cell polarization enriched in lymphoid tissues. Immunology 2005;116:164-171.

24 Nagai M, Usuku K, Matsumoto W, Kodama D, Takenouchi N, Moritoyo T, Hashiguchi S, Ichinose M, Bangham CR, Izumo S, Osame M: Analysis of HTLV-I proviral load in 202 HAM/TSP patients and 243 asymptomatic HTLV-I carriers: high proviral load strongly predisposes to HAM/TSP. J Neurovirol 1998;4:586-593.

25 Jeffery KJ, Usuku K, Hall SE, Matsumoto W, Taylor GP, Procter J, Bunce M, Ogg GS, Welsh KI, Weber JN, Lloyd AL, Nowak MA, Nagai M, Kodama D, Izumo S, Osame M, Bangham CR: HLA alleles determine human T-lymphotropic virus-I (HTLV-I) proviral load and the risk of HTLV-I-associated myelopathy. Proc Natl Acad Sci USA 1999;96:3848-3853.

26 Wattel E, Vartanian JP, Pannetier C, Wain-Hobson S: Clonal expansion of human T-cell leukemia virus type I-infected cells in asymptomatic and symptomatic carriers without malignancy. J Virol 1995;69:2863-2868.

27 Cavrois M, Leclercq I, Gout O, Gessain A, Wain-Hobson S, Wattel E: Persistent oligoclonal expansion of human T-cell leukemia virus type 1-infected circulating cells in patients with tropical spastic paraparesis/HTLV-1 associated myelopathy. 
Oncogene 1998;17:77-82.

28 Yamano Y, Nagai M, Brennan M, Mora CA, Soldan SS, Tomaru U, Takenouchi N, Izumo S, Osame M, Jacobson S: Correlation of human T-cell lymphotropic virus type 1 (HTLV-1) mRNA with proviral DNA load, virus-specific CD8 ${ }^{+} \mathrm{T}$ cells, and disease severity in HTLV-1-associated myelopathy (HAM/TSP). Blood 2002;99:88-94.

29 Asquith B, Mosley AJ, Heaps A, Tanaka Y, Taylor GP, McLean AR, Bangham CR: Quantification of the virus-host interaction in human T lymphotropic virus I infection. Retrovirology DOI: 10.1186/17424690275. 


\section{Figure legends}

Figure 1. Small GTPase pull-down asasay of HTLV-1-infected T cell lines. Bound Rho-, Rac- and Cdc42-GTP was detected in all three HTLV-I-infected T cell lines (HCT-1, HCT-4, and HCT-5); however, bound Rho-, Rac- and Cdc42-GTP were detected in TL-Su, but not in TCL-Kan and C91/PL. In TL-Su, although the amounts of bound Rho- and Rac-GTP seemed almost the same as in cell lines derived from HAM/TSP patients, the amount of bound Cdc42-GTP was obviously lower than in cell lines derived from HAM/TSP patients.

Figure 2. Suppressive effect of HTLV-I production from HTLV-I-infected T cell lines. HTLV-I production from HTLV-I-infected T cell lines derived from HAM/TSP patients (A), but not control cell lines (B), is significantly suppressed by treatment with anti-integrin blocking antibodies. Each cell line was seeded at a concentration of $2 \times 10^{5}$ cells/ml, and cultured in the presence of $20 \mu \mathrm{g} / \mathrm{ml}$ of both anti-CD11a/LFA-1 blocking antibody and anti- $\alpha 4 / C D 49 d$ blocking antibody or $40 \mu \mathrm{g} / \mathrm{ml}$ of control (irrelevant) antibody for 15 hrs. Culture supernatants were collected and the levels of HTLV-I p19 antigen were measured by ELISA. Data are the mean + SD of triplicate cultures. Open bars: control antibody, solid bars: anti-integrin antibodies, N.S.: not significant.

Figure 3. Time course study of the suppressive effect on HTLV-I production from HCT-1 treated with anti- CD11a/LFA-1 and - $\alpha 4 /$ CD49d blocking antibodies. The suppressive effect on HTLV-I production was detected at 3 hrs after the beginning of 
Fukushima, et al. (revised) page 20

culture. Open circle: control antibody, closed square: anti-integrin antibodies.

Figure 4. The effect of treatment with anti- CD11a/LFA-1 and - $\alpha 4 / C D 49 d$ blocking antibodies for HTLV-I tax mRNA expression. Real-time quantitative RT-PCR was performed in a Light-Cycler FastStart DNA Master (Roche Diagnostic, Mannheim, Germany) based on general fluorescence detection with SYBR. HTLV-I tax mRNA expression was evaluated by normalization to $\beta$-actin. There were no significant differences of HTLV-I tax mRNA expression at 6 and 15 hrs after the beginning of culture between treatment with anti- CD11a/LFA-1 and - $\alpha 4 /$ CD49d blocking antibodies and control antibody in HCT-1 and HCT-5. Data are the mean + SD of triplicate cultures. Open bars: control antibody, solid bars: anti-integrin antibodies, N.S.: not significant. 
Figure 1

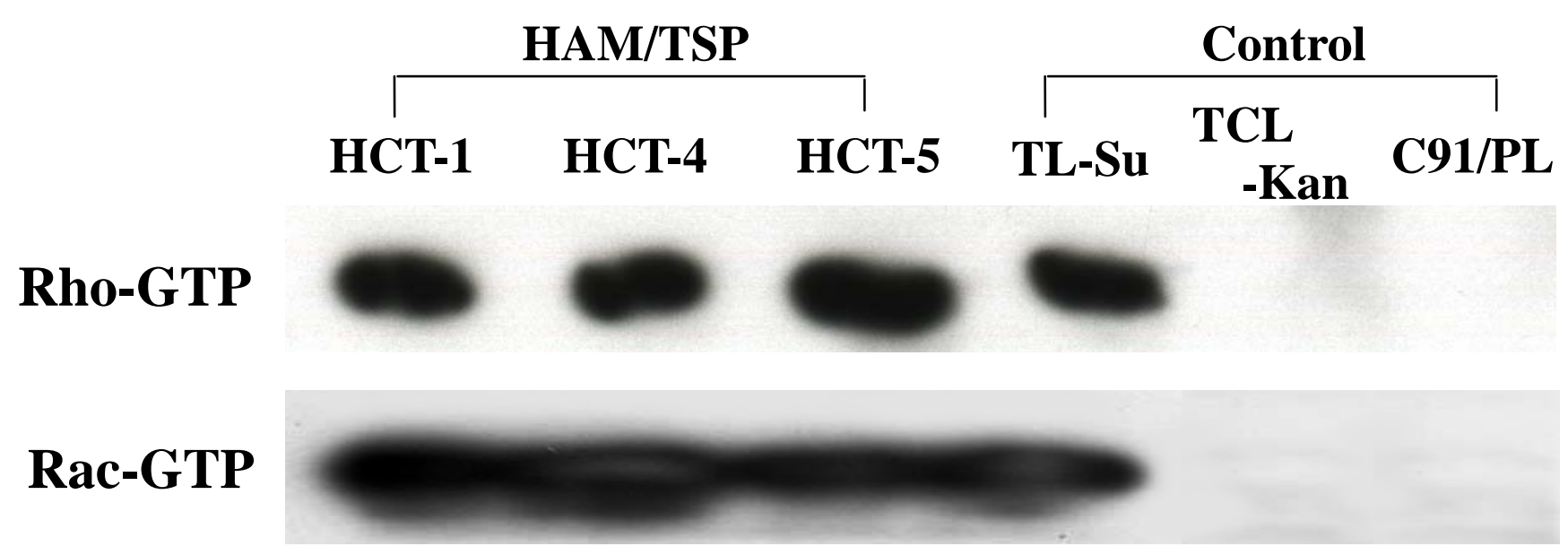

Cdc42-GTP 
Figure 2

(A) HAM/TSP

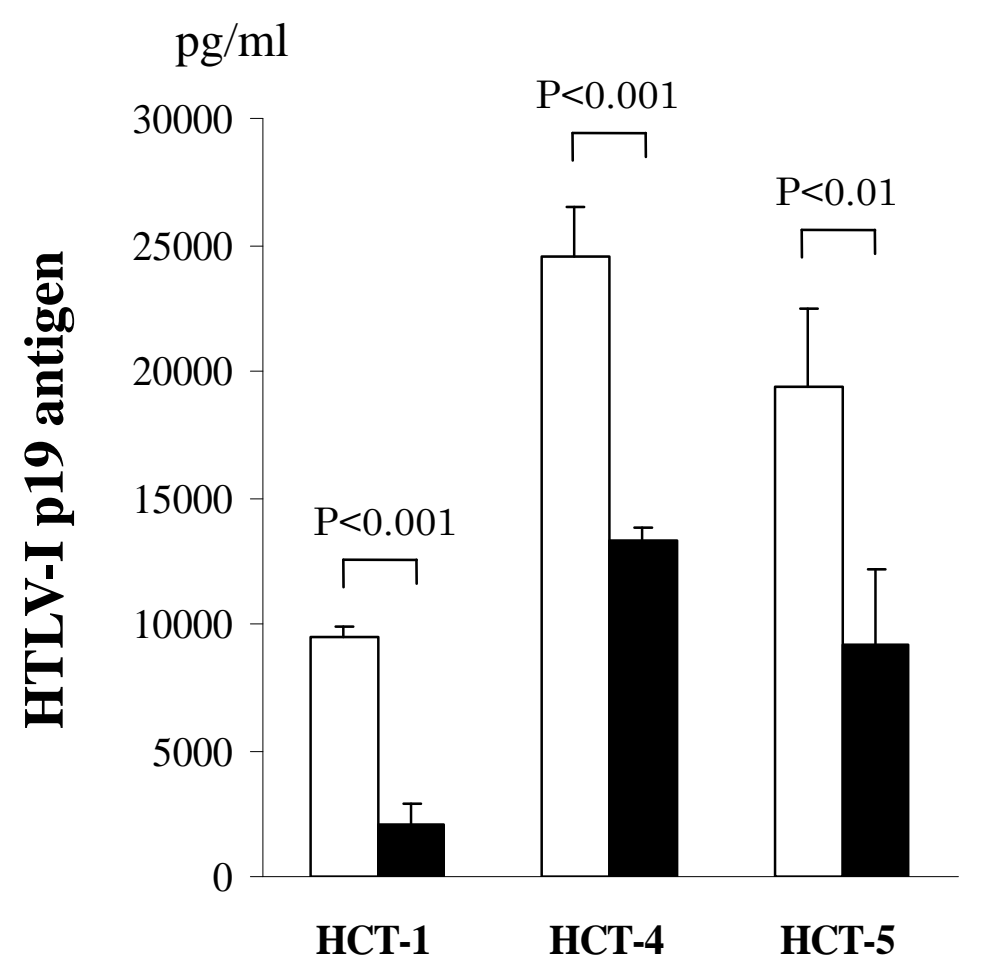

(B) Control

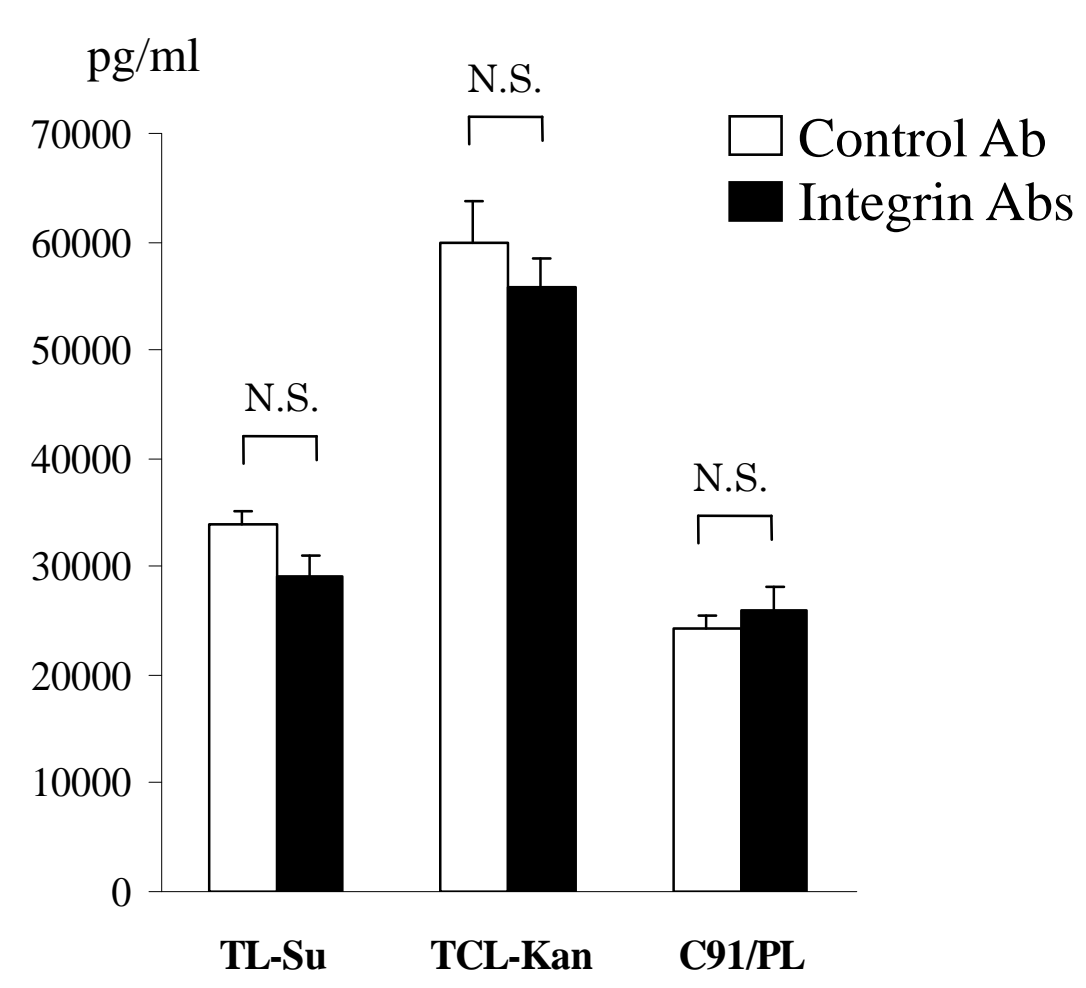


Figure 3

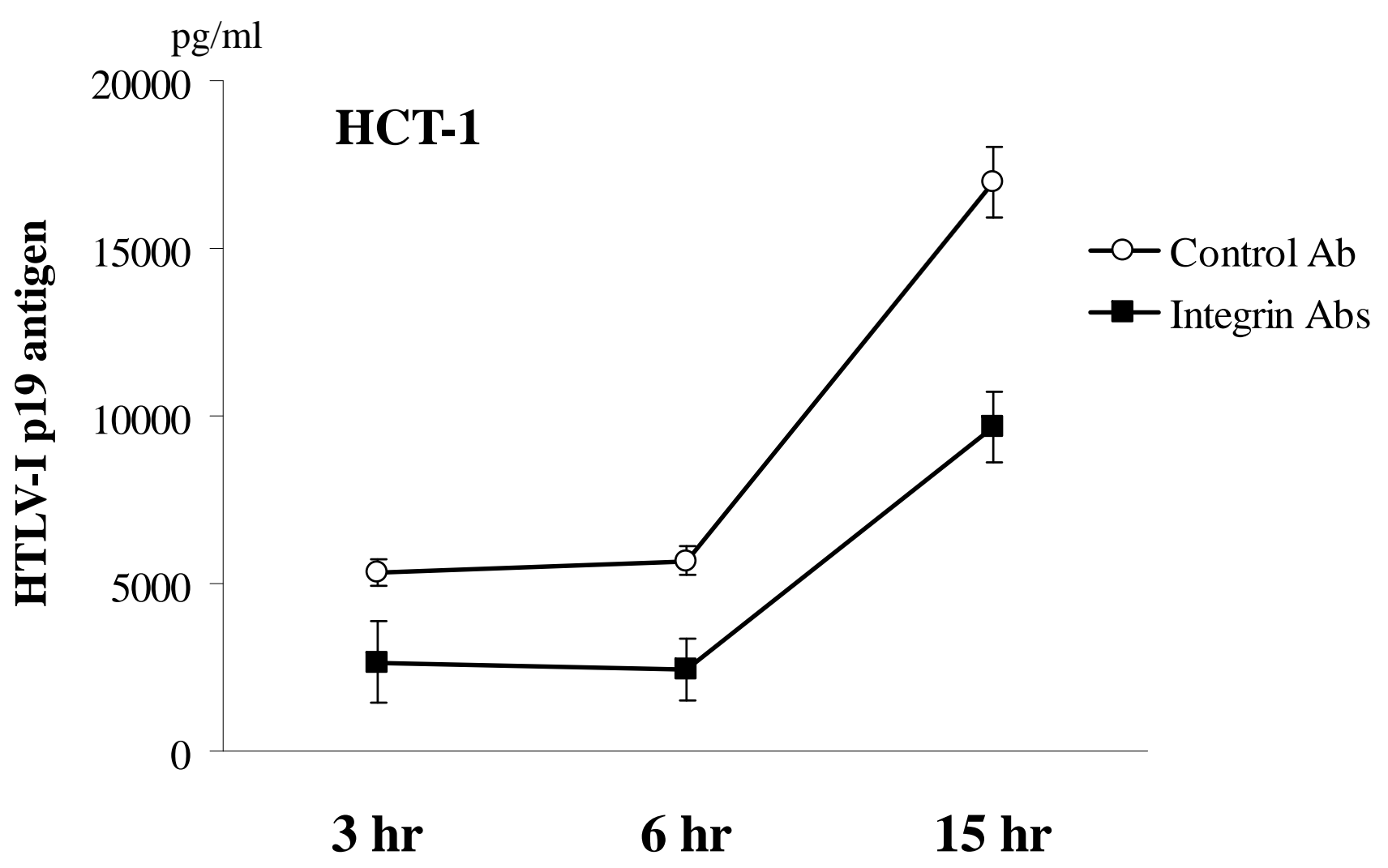


Figure 4

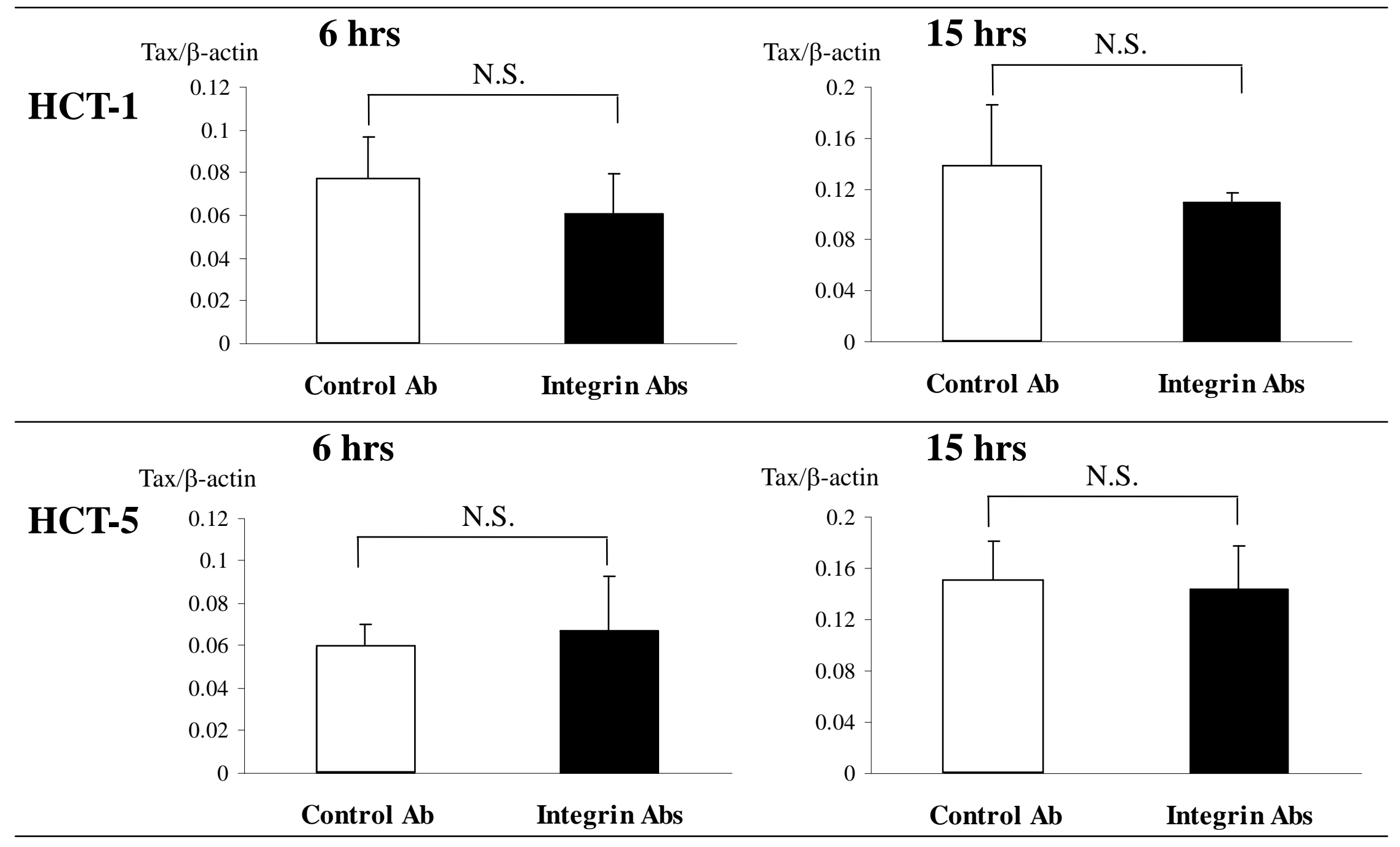

